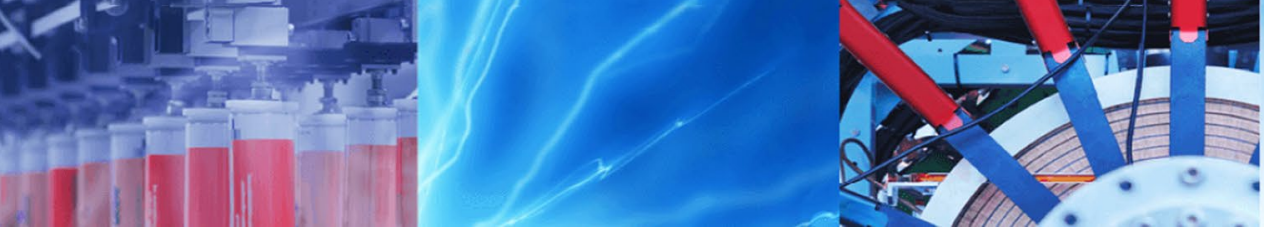

Research Article

\title{
Duality relations of Kamal transform with Laplace, Laplace-Carson, Aboodh, Sumudu, Elzaki, Mohand and Sawi transforms
}

\author{
Sudhanshu Aggarwal ${ }^{1}$ (I) $\cdot$ Nidhi Sharma $^{2} \cdot$ Raman Chauhan $^{2}$
}

Received: 24 September 2019 / Accepted: 11 December 2019 / Published online: 23 December 2019

(c) Springer Nature Switzerland AG 2019

\begin{abstract}
Integral transforms have wide applications in the various disciplines of engineering and science to solve the problems of heat transfer, springs, mixing problems, electrical networks, bending of beams, carbon dating problems, Newton's second law of motion, signal processing, exponential growth and decay problems. In this paper, authors discussed the duality relations of Kamal transform with Laplace, Laplace-Carson, Aboodh, Sumudu, Elzaki, Mohand and Sawi transforms. Tabular presentation of Laplace transform, Laplace-Carson transforms, Aboodh transform, Sumudu transform, Elzaki transform, Mohand transform and Sawi transform of mostly used basic mathematical functions are given to visualize the importance of duality relations of Kamal transform with Laplace, Laplace-Carson, Aboodh, Sumudu, Elzaki, Mohand and Sawi transforms. To show the practical importance of the paper, a problem of the field of pharmacokinetics has been taken and solved in full steps using Kamal transform with the help of mention duality relations. Results show that Kamal transform and Laplace, Laplace-Carson, Aboodh, Sumudu, Elzaki, Mohand and Sawi transforms have a strong relationship.
\end{abstract}

Keywords Laplace $\cdot$ Laplace-Carson · Aboodh · Sumudu · Elzaki $\cdot$ Mohand · Sawi $\cdot$ Kamal transforms

Mathematics Subject Classification $44 \mathrm{~A} 05 \cdot 44 \mathrm{~A} 10 \cdot 44 \mathrm{~A} 15$

\section{Introduction}

Many process and phenomenon of science, engineering and real life can be expressed mathematically and solved by using integral transforms. The problems arise in the field of signal processing, statistics, thermal science, medicine, fractional calculus, aerodynamics, civil engineering, control theory, cardiology, quantum mechanics, space science, marine science, biology, gravitation, nuclear magnetic resonance, heat conduction, economics, telecommunications, nuclear reactors, detection of diabetes, chemistry, stress analysis, electricity, physics, potential theory, mathematics, deflection of beams, vibration of plates, defense, Brownian motion and many other fields can be easily handle with the help of integral transforms by converting them into mathematical form. In the advanced time, researchers are interested in solving the advance problems of research, science, space, engineering and real life by introducing new integral transforms. Aggarwal and Chaudhary [1] discussed Mohand and Laplace transforms comparatively by solving system of differential equations using both integral transforms.

Recently many scholars $[2-6,9]$ used different integral transforms namely Mahgoub (Laplace-Carson) transform, Aboodh transform, Sumudu transform, Elzaki transform, Mohand transform and Kamal transform for evaluating improper integrals which contains error function in the integrand. Mahgoub [7] gave Sawi transform which is a

Sudhanshu Aggarwal, sudhanshu30187@gmail.com; Nidhi Sharma,6nidhisharma@gmail.com; Raman Chauhan, raman28chauhan@

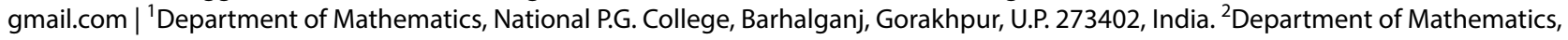
Noida Institute of Engineering and Technology, Greater Noida, U.P. 201306, India. 
new integral transform. Singh and Aggarwal [8] solved the problems of growth and decay by using Sawi transform. Singh and Kumar [10] solved three-dimensional partial differential equations using Wavelet methods. Nazari and Darvishi [11] gave a two dimensional Haar wavelets method for solving system of PDEs. Ali and Baleanu [12] used Haar wavelets for the solution of the problem of unsteady gas flow in four-dimensional. Ali [13] gave the truncation method for solving the time-fractional Benjamin-Ono equation. Ali et al. [14] used HOBW method for the solution of fractional Volterra-Fredholm integrodifferential equations under mixed boundary conditions. Ramadan and Ali [15] gave the approximate solutions for fuzzy Volterra integro-differential equations using HOBB method. Application of Kamal transform for solving linear Volterra integral equations of first kind was given by Aggarwal et al. [16]. Aggarwal et al. [17] used Mohand transform and solved linear Volterra integral equations of second kind. Solution of population growth and decay problems was given by Aggarwal et al. [18] using Mohand transform. Aggarwal et al. [19] gave a new application of Mahgoub transform for finding the solution of linear ordinary differential equations with variable coefficients. Aggarwal and Sharma [20] discussed the application of Kamal transform for solving Abel's integral equation. Watugula [21] defined a new integral transform "Sumudu transform" and used it to solve differential equations and control engineering problems.

The aim of this study is to establish duality relations between Kamal transform and some useful integral transforms namely Laplace transform, Laplace-Carson transform, Aboodh transform, Sumudu transform, Elzaki transform, Mohand transform and Sawi transform.

\section{Laplace transform}

The Laplace transform of the function $Z(\gamma), \gamma \geq 0$ is given by [1]

$L\{Z(\gamma)\}=\int_{0}^{\infty} Z(\gamma) e^{-\epsilon \gamma} d \gamma=B(\epsilon)$.

\section{Laplace-Carson transform}

Laplace-Carson transform of the function $Z(\gamma), \gamma \geq 0$ is given by [2]
$L_{*}\{Z(\gamma)\}=\epsilon \int_{0}^{\infty} Z(\gamma) e^{-\epsilon \gamma} d \gamma=C(\epsilon), \quad 0<k_{1} \leq \epsilon \leq k_{2}$.

\section{Aboodh transform}

Aboodh transform of the function $Z(\gamma), \gamma \geq 0$ is given by [3] $A\{Z(\gamma)\}=\frac{1}{\epsilon} \int_{0}^{\infty} Z(\gamma) e^{-\epsilon \gamma} d \gamma=D(\epsilon), \quad 0<k_{1} \leq \epsilon \leq k_{2}$.

\section{Sumudu transform}

Sumudu transform of the function $Z(\gamma), \gamma \geq 0$ is given by $[4,21]$

$S\{Z(\gamma)\}=\int_{0}^{\infty} Z(\epsilon \gamma) e^{-\gamma} d \gamma=F(\epsilon), \quad 0<k_{1} \leq \epsilon \leq k_{2}$.

\section{Elzaki transform}

Elzaki transform of the function $Z(\gamma), \gamma \geq 0$ is given by [5]

$E\{Z(\gamma)\}=\epsilon \int_{0}^{\infty} Z(\gamma) e^{-\frac{\gamma}{\epsilon}} d \gamma=G(\epsilon), \quad 0<k_{1} \leq \epsilon \leq k_{2}$.

\section{Mohand transform}

Mohand transform of the function $Z(\gamma), \gamma \geq 0$ is given by $[1,6]$

$M\{Z(\gamma)\}=\epsilon^{2} \int_{0}^{\infty} Z(\gamma) e^{-\epsilon \gamma} d \gamma=H(\epsilon), \quad 0<k_{1} \leq \epsilon \leq k_{2}$

\section{Sawi transform}

Sawi transform of the function $Z(\gamma), \gamma \geq 0$ is given by $[7,8]$

$S^{*}\{Z(\gamma)\}=\frac{1}{\epsilon^{2}} \int_{0}^{\infty} Z(\gamma) e^{\frac{-\gamma}{\epsilon}} d \gamma=I(\epsilon), \quad 0<k_{1} \leq \epsilon \leq k_{2}$. 


\section{Kamal transform}

Kamal transform of the function $Z(\gamma), \gamma \geq 0$ is given by [9]

$K\{Z(\gamma)\}=\int_{0}^{\infty} Z(\gamma) e^{\frac{-\gamma}{\epsilon}} d \gamma=J(\epsilon), \quad 0<k_{1} \leq \epsilon \leq k_{2}$.

\section{Dualities of Kamal transform with some useful integral transforms}

In this section, we define the dualities between Kamal transform and some useful integral transforms namely Laplace transform, Laplace-Carson transform, Aboodh transform, Sumudu transform, Elzaki transform, Mohand transform and Sawi transform.

\subsection{Duality between Kamal and Laplace transforms}

If Kamal and Laplace transforms of $Z(\gamma)$ are $J(\epsilon)$ and $B(\epsilon)$ respectively then

$$
J(\epsilon)=B\left(\frac{1}{\epsilon}\right)
$$

and

$B(\epsilon)=J\left(\frac{1}{\epsilon}\right)$

Proof From (8),

$J(\epsilon)=\int_{0}^{\infty} Z(\gamma) e^{\frac{-\gamma}{\epsilon}} d \gamma$

Now, using (1) in above Equation, we obtain

$$
J(\epsilon)=B\left(\frac{1}{\epsilon}\right) \text {. }
$$

To drive (10), we use (1)

$$
B(\epsilon)=\int_{0}^{\infty} Z(\gamma) e^{-\epsilon \gamma} d \gamma
$$

It is immediately concluded using (8) in (11),

$$
B(\epsilon)=J\left(\frac{1}{\epsilon}\right) .
$$

\subsection{Duality between Kamal and Laplace-Carson transforms}

If Kamal and Kamal transforms of $Z(\gamma)$ are $J(\epsilon)$ and $C(\epsilon)$ respectively then

$J(\epsilon)=\epsilon C\left(\frac{1}{\epsilon}\right)$

and

$C(\epsilon)=\epsilon J\left(\frac{1}{\epsilon}\right)$

Proof Using (8) follows

$$
\begin{aligned}
J(\epsilon) & =\int_{0}^{\infty} Z(\gamma) e^{\frac{-\gamma}{\epsilon}} d \gamma \\
& \Rightarrow J(\epsilon)=\epsilon\left[\frac{1}{\epsilon} \int_{0}^{\infty} Z(\gamma) e^{\frac{-\gamma}{\epsilon}} d \gamma\right]
\end{aligned}
$$

Now, using (2) in above equation, we obtain

$J(\epsilon)=\epsilon C\left(\frac{1}{\epsilon}\right)$.

To drive (13), we use (2)

$C(\epsilon)=\epsilon \int_{0}^{\infty} Z(\gamma) e^{-\epsilon \gamma} d \gamma$

It is immediately concluded using (8) in above equation,

$C(\epsilon)=\epsilon J\left(\frac{1}{\epsilon}\right)$.

\subsection{Duality between Kamal and Aboodh transforms}

If Kamal and Aboodh transforms of $Z(\gamma)$ are $J(\epsilon)$ and $D(\epsilon)$ respectively then

$$
J(\epsilon)=\frac{1}{\epsilon} D\left(\frac{1}{\epsilon}\right)
$$

and

$$
D(\epsilon)=\frac{1}{\epsilon} J\left(\frac{1}{\epsilon}\right)
$$

Proof It is immediately concluded from (8) 


$$
\begin{aligned}
J(\epsilon) & =\int_{0}^{\infty} Z(\gamma) e^{\frac{-\gamma}{\epsilon}} d \gamma \\
& \Rightarrow J(\epsilon)=\frac{1}{\epsilon}\left[\epsilon \int_{0}^{\infty} Z(\gamma) e^{\frac{-\gamma}{\epsilon}} d \gamma\right]
\end{aligned}
$$

Now, using (3) in above Equation, we have

$J(\epsilon)=\frac{1}{\epsilon} D\left(\frac{1}{\epsilon}\right)$.

To drive (16), we use (3)

$D(\epsilon)=\frac{1}{\epsilon} \int_{0}^{\infty} Z(\gamma) e^{-\epsilon \gamma} d \gamma$

It is immediately concluded using (8) in above equation, $D(\epsilon)=\frac{1}{\epsilon} J\left(\frac{1}{\epsilon}\right)$.

\subsection{Duality between Kamal and Sumudu transforms}

If Kamal and Sumudu transforms of $Z(\gamma)$ are $J(\epsilon)$ and $F(\epsilon)$ respectively then

$J(\epsilon)=\epsilon F(\epsilon)$

and

$F(\epsilon)=\frac{1}{\epsilon} J(\epsilon)$

Proof From (8), we have

$J(\epsilon)=\int_{0}^{\infty} Z(\gamma) e^{\frac{-\gamma}{\epsilon}} d \gamma$

Put $\frac{\gamma}{\epsilon}=u \Rightarrow d \gamma=\epsilon d u$ in above equation, we have

$$
\begin{aligned}
J(\epsilon) & =\int_{0}^{\infty} Z(\epsilon u) e^{-u} \epsilon d u \\
& \Rightarrow J(\epsilon)=\epsilon \int_{0}^{\infty} Z(\epsilon u) e^{-u} d u
\end{aligned}
$$

Now, using (4) in above equation, we have $J(\epsilon)=\epsilon F(\epsilon)$.

To drive (18), we use (4)

$$
\begin{aligned}
& F(\epsilon)=\int_{0}^{\infty} Z(\epsilon \gamma) e^{-\gamma} d \gamma \\
& \text { Put } \epsilon \gamma=u \Rightarrow d \gamma=\frac{d u}{\epsilon} \text { in above equation, we have } \\
& F(\epsilon)=\int_{0}^{\infty} Z(u) e^{-\frac{u}{\epsilon}} \frac{d u}{\epsilon} \\
& \Rightarrow F(\epsilon)=\frac{1}{\epsilon} \int_{0}^{\infty} Z(u) e^{-\frac{u}{\epsilon}} d u
\end{aligned}
$$

It is immediately concluded using (8) in above equation, $F(\epsilon)=\frac{1}{\epsilon} J(\epsilon)$

\subsection{Duality between Kamal and Elzaki transforms}

If Kamal and Elzaki transforms of $Z(\gamma)$ are $J(\epsilon)$ and $G(\epsilon)$ respectively then

$$
J(\epsilon)=\frac{1}{\epsilon} G(\epsilon)
$$

and

$$
G(\epsilon)=\epsilon J(\epsilon)
$$

Proof From (8), we have

$$
\begin{aligned}
J(\epsilon) & =\int_{0}^{\infty} Z(\gamma) e^{\frac{-\gamma}{\epsilon}} d \gamma \\
& \Rightarrow J(\epsilon)=\frac{1}{\epsilon}\left[\epsilon \int_{0}^{\infty} Z(\gamma) e^{\frac{-\gamma}{\epsilon}} d \gamma\right]
\end{aligned}
$$

Now, using (5) in above equation, we have

$J(\epsilon)=\frac{1}{\epsilon} G(\epsilon)$.

To drive (20), we use (5)

$G(\epsilon)=\epsilon \int_{0}^{\infty} Z(\gamma) e^{-\frac{\gamma}{\epsilon}} d \gamma$

It is immediately concluded using (8) in above equation, $G(\epsilon)=\epsilon J(\epsilon)$. 


\subsection{Duality between Kamal and Mohand transforms}

If Kamal and Mohand transforms of $Z(\gamma)$ are $J(\epsilon)$ and $H(\epsilon)$ respectively then

$J(\epsilon)=\epsilon^{2} H\left(\frac{1}{\epsilon}\right)$

and

$H(\epsilon)=\epsilon^{2} J\left(\frac{1}{\epsilon}\right)$

Proof From (8), we have

$$
\begin{aligned}
J(\epsilon) & =\int_{0}^{\infty} Z(\gamma) e^{\frac{-\gamma}{\epsilon}} d \gamma \\
& \Rightarrow J(\epsilon)=\epsilon^{2}\left[\frac{1}{\epsilon^{2}} \int_{0}^{\infty} Z(\gamma) e^{\frac{-\gamma}{\epsilon}} d \gamma\right]
\end{aligned}
$$

Now, using (6) in above equation, we have

$$
J(\epsilon)=\epsilon^{2} H\left(\frac{1}{\epsilon}\right) \text {. }
$$

To drive (22), we use (6)

$$
H(\epsilon)=\epsilon^{2} \int_{0}^{\infty} Z(\gamma) e^{-\epsilon \gamma} d \gamma
$$

It is immediately concluded using (8) in above equation, $H(\epsilon)=\epsilon^{2} J\left(\frac{1}{\epsilon}\right)$.

\subsection{Duality between Kamal and Sawi transforms}

If Kamal and Sawi transforms of $Z(\gamma)$ are $J(\epsilon)$ and $I(\epsilon)$ respectively then

$J(\epsilon)=\epsilon^{2} I(\epsilon)$

and

$$
I(\epsilon)=\frac{1}{\epsilon^{2}} J(\epsilon)
$$

Proof Using (8) follows

$$
\begin{aligned}
J(\epsilon) & =\int_{0}^{\infty} Z(\gamma) e^{\frac{-\gamma}{\epsilon}} d \gamma \\
& \Rightarrow J(\epsilon)=\epsilon^{2}\left[\frac{1}{\epsilon^{2}} \int_{0}^{\infty} Z(\gamma) e^{\frac{-\gamma}{\epsilon}} d \gamma\right]
\end{aligned}
$$

Now, using (7) in above equation, we obtain

$$
J(\epsilon)=\epsilon^{2} l(\epsilon) \text {. }
$$

To drive (24), we use (7)

$$
I(\epsilon)=\frac{1}{\epsilon^{2}} \int_{0}^{\infty} Z(\gamma) e^{-\frac{\gamma}{\epsilon}} d \gamma
$$

Now, using (8) in above equation, we obtain

$$
I(\epsilon)=\frac{1}{\epsilon^{2}} J(\epsilon)
$$

\section{Applications of mention duality relations for finding integral transforms (Laplace transform, Laplace-Carson transform, Aboodh transform, Sumudu transform, Elzaki transform, Mohand transform and Sawi transform) of useful basic functions}

We are giving tabular presentation of the integral transforms of mostly used basic functions by using mention dualities relations to visualize the usefulness of dualities between Kamal transform and mention integral transforms in the application field (Tables 1, 2, 3, 4, 5, 6, 7). 
Table 1 Laplace transform of useful basic functions with the help of relation of duality between Kamal and Laplace transforms

\begin{tabular}{llll}
\hline S. no. & $Z(\gamma)$ & $K\{Z(\gamma)\}=J(\epsilon)$ & $L\{Z(\gamma)\}=B(\epsilon)$ \\
\hline 1. & 1 & $\epsilon$ & $\frac{1}{\epsilon}$ \\
2. & $\gamma$ & $\epsilon^{2}$ & $\frac{1}{\epsilon^{2}}$ \\
3. & $\gamma^{2}$ & $2 ! \epsilon^{3}$ & $\frac{2 !}{\epsilon^{3}}$ \\
4. & $\gamma^{n}$, & $n ! \epsilon^{n+1}$ & $\frac{n !}{\epsilon^{n+1}}$ \\
5. & $n \in N$ & & $\frac{\Gamma(n+1)}{\epsilon^{n+1}}$ \\
6. & $\gamma^{n}, n>-1$ & $\Gamma(n+1) \epsilon^{n+1}$ & $\frac{1}{(\epsilon-a)}$ \\
7. & $e^{a \gamma}$ & $\frac{\epsilon}{(1-a \epsilon)}$ & $\frac{a}{\left(\epsilon^{2}+a^{2}\right)}$ \\
8. & $\sin a \gamma$ & $\frac{a \epsilon^{2}}{\left(1+a^{2} \epsilon^{2}\right)}$ & $\frac{\epsilon}{\left(\epsilon^{2}+a^{2}\right)}$ \\
9. & $\cos a \gamma$ & $\frac{\epsilon}{\left(1+a^{2} \epsilon^{2}\right)}$ & $\frac{a}{\left(\epsilon^{2}-a^{2}\right)}$ \\
10. & $\sinh a \gamma$ & $\frac{a \epsilon^{2}}{\left(1-a^{2} \epsilon^{2}\right)}$ & $\frac{\epsilon}{\left(\epsilon^{2}-a^{2}\right)}$ \\
\hline
\end{tabular}

Table 2 Laplace-Carson transform of useful basic functions with the help of relation of duality between Kamal and Laplace-Carson transforms

\begin{tabular}{|c|c|c|c|}
\hline S. no. & $Z(\gamma)$ & $K\{Z(\gamma)\}=J(\epsilon)$ & $L_{*}\{Z(\gamma)\}=C(\epsilon)$ \\
\hline 1. & 1 & $\epsilon$ & 1 \\
\hline 2. & $\gamma$ & $\epsilon^{2}$ & $\frac{1}{6}$ \\
\hline 3. & $\gamma^{2}$ & $2 ! \epsilon^{3}$ & $\frac{2 !}{\epsilon^{2}}$ \\
\hline 4. & $\begin{array}{l}\gamma^{n}, \\
n \in N\end{array}$ & $n ! \epsilon^{n+1}$ & $\frac{n !}{\epsilon^{n}}$ \\
\hline 5. & $\gamma^{n}, n>-1$ & $\Gamma(n+1) \epsilon^{n+1}$ & $\frac{\Gamma(n+1)}{\epsilon^{n}}$ \\
\hline 6. & $e^{a \gamma}$ & $\frac{\epsilon}{(1-a \epsilon)}$ & $\frac{\epsilon}{(\epsilon-a)}$ \\
\hline 7. & $\sin a \gamma$ & $\frac{a \epsilon^{2}}{\left(1+a^{2} \epsilon^{2}\right)}$ & $\frac{a \epsilon}{\left(\epsilon^{2}+a^{2}\right)}$ \\
\hline 8. & $\cos a \gamma$ & $\frac{\epsilon}{\left(1+a^{2} \epsilon^{2}\right)}$ & $\frac{\epsilon^{2}}{\left(\epsilon^{2}+a^{2}\right)}$ \\
\hline 9. & sinhar & $\frac{a \epsilon^{2}}{\left(1-a^{2} \epsilon^{2}\right)}$ & $\frac{a \epsilon}{\left(\epsilon^{2}-a^{2}\right)}$ \\
\hline 10. & coshar & $\frac{\epsilon}{\left(1-a^{2} \epsilon^{2}\right)}$ & $\frac{\epsilon^{2}}{\left(\epsilon^{2}-a^{2}\right)}$ \\
\hline
\end{tabular}

Table 3 Aboodh transform of useful basic functions with the help of relation of duality between Kamal and Aboodh transforms

\begin{tabular}{|c|c|c|c|}
\hline S. no. & $Z(\gamma)$ & $K\{Z(\gamma)\}=J(\epsilon)$ & $A\{Z(\gamma)\}=D(\epsilon)$ \\
\hline 1. & 1 & $\epsilon$ & $\frac{1}{c^{2}}$ \\
\hline 2. & $\gamma$ & $\epsilon^{2}$ & $\frac{\epsilon^{2}}{\epsilon^{3}}$ \\
\hline 3. & $\gamma^{2}$ & $2 ! \epsilon^{3}$ & $\begin{array}{l}\epsilon^{3} \\
\frac{2 !}{\epsilon^{4}}\end{array}$ \\
\hline 4. & $\gamma^{n}, n \in N$ & $n ! \epsilon^{n+1}$ & $\frac{n !}{\epsilon^{n+2}}$ \\
\hline 5. & $\gamma^{n}, n>-1$ & $\Gamma(n+1) \epsilon^{n+1}$ & $\frac{\Gamma(n+1)}{\epsilon^{n+2}}$ \\
\hline 6. & $e^{a \gamma}$ & $\frac{\epsilon}{(1-a \epsilon)}$ & $\frac{1}{\epsilon(\epsilon-a)}$ \\
\hline 7. & $\sin a \gamma$ & $\frac{a \epsilon^{2}}{\left(1+a^{2} \epsilon^{2}\right)}$ & $\frac{a}{\epsilon\left(\epsilon^{2}+a^{2}\right)}$ \\
\hline 8. & $\cos a \gamma$ & $\frac{\epsilon}{\left(1+a^{2} \epsilon^{2}\right)}$ & $\frac{1}{\left(\epsilon^{2}+a^{2}\right)}$ \\
\hline 9. & sinhar & $\frac{a \epsilon^{2}}{\left(1-a^{2} \epsilon^{2}\right)}$ & $\frac{a}{\epsilon\left(\epsilon^{2}-a^{2}\right)}$ \\
\hline 10. & coshar & $\frac{\epsilon}{\left(1-a^{2} \epsilon^{2}\right)}$ & $\frac{1}{\left(\epsilon^{2}-a^{2}\right)}$ \\
\hline
\end{tabular}

Table 4 Sumudu transform of useful basic functions with the help of relation of duality between Kamal and Sumudu transforms

\begin{tabular}{|c|c|c|c|}
\hline S. no. & $Z(\gamma)$ & $K\{Z(\gamma)\}=J(\epsilon)$ & $S\{Z(\gamma)\}=F(\epsilon)$ \\
\hline 1. & 1 & $\epsilon$ & 1 \\
\hline 2. & $\gamma$ & $\epsilon^{2}$ & $\epsilon$ \\
\hline 3. & $\gamma^{2}$ & $2 ! \epsilon^{3}$ & $2 ! \epsilon^{2}$ \\
\hline 4. & $\gamma^{n}, n \in N$ & $n ! \epsilon^{n+1}$ & $n ! \epsilon^{n}$ \\
\hline 5. & $\gamma^{n}, n>-1$ & $\Gamma(n+1) \epsilon^{n+1}$ & $\Gamma(n+1) \epsilon^{n}$ \\
\hline 6. & $e^{a \gamma}$ & $\frac{\epsilon}{(1-a \epsilon)}$ & $\frac{1}{(1-a \epsilon)}$ \\
\hline 7. & $\sin a \gamma$ & $\frac{a \epsilon^{2}}{\left(1+a^{2} \epsilon^{2}\right)}$ & $\frac{a \epsilon}{\left(1+a^{2} \epsilon^{2}\right)}$ \\
\hline 8. & $\cos a \gamma$ & $\frac{\epsilon}{\left(1+a^{2} \epsilon^{2}\right)}$ & $\frac{1}{\left(1+a^{2} \epsilon^{2}\right)}$ \\
\hline 9. & sinhar & $\frac{a \epsilon^{2}}{\left(1-a^{2} \epsilon^{2}\right)}$ & $\frac{a \epsilon}{\left(1-a^{2} \epsilon^{2}\right)}$ \\
\hline 10. & coshar & $\frac{\epsilon}{\left(1-a^{2} \epsilon^{2}\right)}$ & $\frac{1}{\left(1-a^{2} \epsilon^{2}\right)}$ \\
\hline
\end{tabular}

Table 5 Elzaki transform of useful basic functions with the help of relation of duality between Kamal and Elzaki transforms

\begin{tabular}{|c|c|c|c|}
\hline S. no. & $Z(\gamma)$ & $K\{Z(\gamma)\}=J(\epsilon)$ & $E\{Z(\gamma)\}=G(\epsilon)$ \\
\hline 1. & 1 & $\epsilon$ & $\epsilon^{2}$ \\
\hline 2. & $\gamma$ & $\epsilon^{2}$ & $\epsilon^{3}$ \\
\hline 3. & $\gamma^{2}$ & $2 ! \epsilon^{3}$ & $2 ! \epsilon^{4}$ \\
\hline 4. & $\gamma^{n}, n \in N$ & $n ! \epsilon^{n+1}$ & $n ! \epsilon^{n+2}$ \\
\hline 5. & $\gamma^{n}, n>-1$ & $\Gamma(n+1) \epsilon^{n+1}$ & $\Gamma(n+1) \epsilon^{n+2}$ \\
\hline 6. & $e^{a \gamma}$ & $\frac{\epsilon}{(1-a \epsilon)}$ & $\frac{\epsilon^{2}}{(1-a \epsilon)}$ \\
\hline 7. & $\sin a \gamma$ & $\frac{a \epsilon^{2}}{\left(1+a^{2} \epsilon^{2}\right)}$ & $\frac{a \epsilon^{3}}{\left(1+a^{2} \epsilon^{2}\right)}$ \\
\hline 8. & $\cos a \gamma$ & $\frac{\epsilon}{\left(1+a^{2} \epsilon^{2}\right)}$ & $\frac{\epsilon^{2}}{\left(1+a^{2} \epsilon^{2}\right)}$ \\
\hline 9. & sinhar & $\frac{a \epsilon^{2}}{\left(1-a^{2} \epsilon^{2}\right)}$ & $\frac{a \epsilon^{3}}{\left(1-a^{2} \epsilon^{2}\right)}$ \\
\hline 10. & coshar & $\frac{\epsilon}{\left(1-a^{2} \epsilon^{2}\right)}$ & $\frac{\epsilon^{2}}{\left(1-a^{2} \epsilon^{2}\right)}$ \\
\hline
\end{tabular}


Table 6 Mohand transform of useful basic functions with the help of relation of duality between Kamal and Mohand transforms

\begin{tabular}{|c|c|c|c|}
\hline S. no. & $Z(\gamma)$ & $K\{Z(\gamma)\}=J(\epsilon)$ & $M\{Z(\gamma)\}=H(\epsilon)$ \\
\hline 1. & 1 & $\epsilon$ & $\epsilon$ \\
\hline 2. & $\gamma$ & $\epsilon^{2}$ & 1 \\
\hline 3. & $\gamma^{2}$ & $2 ! \epsilon^{3}$ & $\frac{2 !}{\epsilon}$ \\
\hline 4. & $\gamma^{n}, n \in N$ & $n ! \epsilon^{n+1}$ & $\frac{n !}{\epsilon^{n-1}}$ \\
\hline 5. & $\gamma^{n}, n>-1$ & $\Gamma(n+1) \epsilon^{n+1}$ & $\frac{\Gamma(n+1)}{\epsilon^{n-1}}$ \\
\hline 6. & $e^{a \gamma}$ & $\frac{\epsilon}{(1-a \epsilon)}$ & $\frac{\epsilon^{2}}{(\epsilon-a)}$ \\
\hline 7. & $\sin a \gamma$ & $\frac{a \epsilon^{2}}{\left(1+a^{2} \epsilon^{2}\right)}$ & $\frac{a \epsilon^{2}}{\left(\epsilon^{2}+a^{2}\right)}$ \\
\hline 8. & $\cos a \gamma$ & $\frac{\epsilon}{\left(1+a^{2} \epsilon^{2}\right)}$ & $\frac{\epsilon^{3}}{\left(\epsilon^{2}+a^{2}\right)}$ \\
\hline 9. & sinhar & $\frac{a \epsilon^{2}}{\left(1-a^{2} \epsilon^{2}\right)}$ & $\frac{a \epsilon^{2}}{\left(\epsilon^{2}-a^{2}\right)}$ \\
\hline 10. & coshar & $\frac{\epsilon}{\left(1-a^{2} \epsilon^{2}\right)}$ & $\frac{\epsilon^{3}}{\left(\epsilon^{2}-a^{2}\right)}$ \\
\hline
\end{tabular}

Table 7 Sawi transform of useful basic functions with the help of relation of duality between Kamal and Sawi transforms

\begin{tabular}{llll}
\hline S.n. & $Z(\gamma)$ & $K\{Z(\gamma)\}=J(\epsilon)$ & $S^{*}\{Z(\gamma)\}=I(\epsilon)$ \\
\hline 1. & 1 & $\epsilon$ & $\frac{1}{\epsilon}$ \\
2. & $\gamma$ & $\epsilon^{2}$ & 1 \\
3. & $\gamma^{2}$ & $2 ! \epsilon^{3}$ & $2 ! \epsilon$ \\
4. & $\gamma^{n}, n \in N$ & $n ! \epsilon^{n+1}$ & $n ! \epsilon^{n-1}$ \\
5. & $\gamma^{n}, n>-1$ & $\Gamma(n+1) \epsilon^{n+1}$ & $\Gamma(n+1) \epsilon^{n-1}$ \\
6. & $e^{a \gamma}$ & $\frac{\epsilon}{(1-a \epsilon)}$ & $\frac{1}{\epsilon(1-a)}$ \\
7. & $\sin a \gamma$ & $\frac{a \epsilon^{2}}{\left(1+a^{2} \epsilon^{2}\right)}$ & $\frac{a}{\left(1+a^{2} \epsilon^{2}\right)}$ \\
8. & $\cos a \gamma$ & $\frac{\epsilon}{\left(1+a^{2} \epsilon^{2}\right)}$ & $\frac{1}{\epsilon\left(1+a^{2} \epsilon^{2}\right)}$ \\
9. & $\sinh a \gamma$ & $\frac{a \epsilon^{2}}{\left(1-a^{2} \epsilon^{2}\right)}$ & $\frac{a}{\left(1-a^{2} \epsilon^{2}\right)}$ \\
10. & $\cosh a \gamma$ & $\frac{\epsilon}{\left(1-a^{2} \epsilon^{2}\right)}$ & $\frac{1}{\epsilon\left(1-a^{2} \epsilon^{2}\right)}$ \\
\hline
\end{tabular}

\section{Kamal transforms of the derivatives using Kamal-Laplace duality relation}

In this section, Kamal transforms of the derivatives of the function $Z(\gamma)$ are given with the help of Kamal-Laplace duality relation.

Laplace transforms of the derivatives of the function $Z(\gamma)$ are given by [1]

$L\left\{Z^{\prime}(\gamma)\right\}=\epsilon L\{Z(\gamma)\}-Z(0)$

$L\left\{Z^{\prime \prime}(\gamma)\right\}=\epsilon^{2} L\{Z(\gamma)\}-\epsilon Z(0)-Z^{\prime}(0)$
$L\left\{Z^{(n)}(\gamma)\right\}=\epsilon^{n} L\{Z(\gamma)\}-\epsilon^{n-1} Z(0)-\epsilon^{n-2} Z^{\prime}(0)$

$$
-\cdots-\epsilon Z^{(n-2)}(0)-Z^{(n-1)}(0)
$$

Using (9) (Kamal-Laplace duality relation) on (25), (26) and (27), we have

$$
\begin{aligned}
K\left\{Z^{\prime}(\gamma)\right\}= & \frac{1}{\epsilon} K\{Z(\gamma)\}-Z(0) \\
K\left\{Z^{\prime \prime}(\gamma)\right\}= & \frac{1}{\epsilon^{2}} K\{Z(\gamma)\}-\frac{1}{\epsilon} Z(0)-Z^{\prime}(0) \\
K\left\{Z^{(n)}(\gamma)\right\}= & \frac{1}{\epsilon^{n}} K\{Z(\gamma)\}-\frac{1}{\epsilon^{n-1}} Z(0)-\frac{1}{\epsilon^{n-2}} Z^{\prime}(0) \\
& -\cdots-\frac{1}{\epsilon} Z^{(n-2)}(0)-Z^{(n-1)}(0)
\end{aligned}
$$

\section{Solution of the problem of pharmacokinetics with the help of mention duality relations}

For physical explanation of the present scheme, we consider a problem from the field of pharmacokinetics for finding the concentration of drug at any given time in the blood during continuous intravenous injection of drug and find its solution in this section with the help of discussed duality relations between Kamal transform and other integral transforms. Mathematically, this problem can be expressed in terms of first order linear differential equation with initial condition as

$\frac{d C(t)}{d t}+\alpha C(t)=\frac{\beta}{V_{o l}}, t>0$

with

$C(0)=0$

Here $C(t)$ : drug concentration in the blood at any time $t \alpha$ : constant velocity of elimination $\beta$ : the rate of infusion (in $\mathrm{mg} / \mathrm{min}$ ) Vol: volume in which drug is distributed.

Kamal transform of both sides of (31) gives

$K\left\{\frac{d C(t)}{d t}\right\}+\alpha K\{C(t)\}=\frac{\beta}{V o l} K\{1\}$

Applying (28) on (33), we get

$\frac{1}{\epsilon} K\{C(t)\}-C(0)+\alpha K\{C(t)\}=\frac{\beta}{V o l} \epsilon$

The use of (32) in (34) gives 
$K\{C(t)\}=\frac{\beta}{V o l}\left(\frac{\epsilon^{2}}{1+\alpha \epsilon}\right)$

Applying inverse Kamal transform on (35), we have

$$
\begin{aligned}
C(t) & =\frac{\beta}{V o l} K^{-1}\left\{\frac{\epsilon^{2}}{1+\alpha \epsilon}\right\}=\frac{\beta}{V o l} K^{-1}\left\{\frac{1}{\alpha}\left(\epsilon-\frac{\epsilon}{1+\alpha \epsilon}\right)\right\} \\
& \Rightarrow C(t)=\frac{\beta}{\alpha V_{o l}}\left[K^{-1}\{\epsilon\}-K^{-1}\left\{\frac{\epsilon}{1+\alpha \epsilon}\right\}\right] \\
& \Rightarrow C(t)=\frac{\beta}{\alpha V_{o l}}\left[1-e^{-\alpha t}\right]
\end{aligned}
$$

which is the required concentration of drug at any given time in the blood during continuous intravenous injection of drug.

\section{Conclusions}

In the present paper, duality relations between Kamal transform and some useful integral transforms namely Laplace transform, Laplace-Carson transform, Aboodh transform, Sumudu transform, Elzaki transform, Mohand transform and Sawi transform are established successfully. Tabular presentation of the integral transforms (Laplace transform, Laplace-Carson transform, Aboodh transform, Sumudu transform, Elzaki transform, Mohand transform and Sawi transform) of mostly used basic functions are given with the help of mention dualities relations to visualize the importance of dualities between Kamal transform and mention integral transforms. For physical explanation of the paper, a problem from the field of pharmacokinetics is taken and solved in full steps using Kamal transform with the help of mention duality relations. Results show that the Kamal transform and mention integral transforms in this paper are strongly related to each others. In future using these duality relations, we can easily solved many advanced problems of modern era such as motion of coupled harmonic oscillators, drug distribution in the body, arms race models, Brownian motion and the common health problem such as detection of diabetes.

\section{Compliance with ethical standards}

Conflict of interest All authors states that they have no conflict of interest.

\section{References}

1. Aggarwal S, Chaudhary R (2019) A comparative study of Mohand and Laplace transforms. J Emerg Technol Innov Res 6(2):230-240

2. Aggarwal S, Gupta AR, Sharma SD, Chauhan R, Sharma N (2019) Mahgoub transform (Laplace-Carson transform) of error function. Int J Latest Technol Eng Manag Appl Sci 8(4):92-98

3. Aggarwal S, Singh GP (2019) Aboodh transform of error function. Univ Rev 10(6):137-150

4. Aggarwal S, Sharma SD (2019) Sumudu transform of error function. J Appl Sci Comput 6(6):1222-1231

5. Aggarwal S, Gupta AR, Kumar A (2019) Elzaki transform of error function. Glob J Eng Sci Res 6(5):412-422

6. Aggarwal S, Gupta AR, Kumar D (2019) Mohand transform of error function. Int J Res Advent Technol 7(5):224-231

7. Mahgoub MMA (2019) The new integral transform "Sawi Transform". Adv Theor Appl Math 14(1):81-87

8. Singh GP, Aggarwal S (2019) Sawi transform for population growth and decay problems. Int J Latest Technol Eng Manag Appl Sci 8(8):157-162

9. Aggarwal S, Singh GP (2019) Kamal transform of error function. J Appl Sci Comput 6(5):2223-2235

10. Singh I, Kumar S (2017) Wavelet methods for solving three-dimensional partial differential equations. Math Sci 11(2):145-154

11. Nazari AA, Darvishi MT (2017) A two dimensional Haar wavelets method for solving system of PDEs. Appl Math Comput 292:33-46

12. Ali MR, Baleanu D (2019) Haar wavelets scheme for solving the unsteady gas flow in four-dimensional. Therm Sci 23:292-301

13. Ali MR (2019) A truncation method for solving the time-fractional Benjamin-Ono equation. J Appl Math 18:1-7

14. Ali MR, Hadhoud AR, Srivastava HM (2019) Solution of fractional Volterra-Fredholm integro-differential equations under mixed boundary conditions by using the HOBW method. Adv Differ Equ 2019:1-14

15. Ramadan MA, Ali MR (2017) Approximate solutions for fuzzy Volterra integro-differential equations using HOBB method. J Stat Appl Probab 4(14):80-94

16. Aggarwal S, Sharma N, Chauhan R (2018) Application of Kamal transform for solving linear Volterra integral equations of first kind. Int J Res Advent Technol 6(8):2081-2088

17. Aggarwal S, Sharma N, Chauhan R (2018) Solution of linear Volterra integral equations of second kind using Mohand transform. Int J Res Advent Technol 6(11):3098-3102

18. Aggarwal S, Sharma N, Chauhan R (2018) Solution of population growth and decay problems by using Mohand transform. Int J Res Advent Technol 6(11):3277-3282

19. Aggarwal S, Sharma N, Chauhan R, Gupta AR, Khandelwal A (2018) A new application of Mahgoub transform for solving linear ordinary differential equations with variable coefficients. J Comput Math Sci 9(6):520-525

20. Aggarwal S, Sharma SD (2019) Application of Kamal transform for solving Abel's integral equation. Glob J Eng Sci Res 6(3):82-90

21. Watugula GK (1993) Sumudu transform: a new integral transform to solve differential equations and control engineering problems. Int J Math Educ Sci Technol 24(1):35-43

Publisher's Note Springer Nature remains neutral with regard to jurisdictional claims in published maps and institutional affiliations. 